\title{
Preparation of 2,5-Bis(Aminomethyl)Furan by Direct Reductive Amination of 2,5-Diformylfuran over Nickel-Raney Catalysts
}

\author{
Ngoc-Thuc Le',2, Areum Byun1, Yohan Han 1,2, Kee-In Lee',2, Hyungrok Kim1,2* \\ ${ }^{1}$ Center for Nanocatalysts, Green Chemistry and Engineering Division, Korea Research Institute of Chemical \\ Technology (KRICT), Daejeon, Korea \\ ${ }^{2}$ Department of Green Chemistry and Environmental Biotechnology, Korea University of Science and \\ Technology (UST), Daejeon, Korea \\ Email:
}

Received 23 June 2015; accepted 2 August 2015; published 5 August 2015

Copyright (c) 2015 by authors and Scientific Research Publishing Inc.

This work is licensed under the Creative Commons Attribution International License (CC BY).

http://creativecommons.org/licenses/by/4.0/

(c) (i) Open Access

\section{Abstract}

The direct reductive amination of 2,5-diformylfuran (DFF) with ammonia to 2,5-bis(aminomethyl)furan (BAF) was demonstrated, for the first time, over the commercial type Nickel-Raney and acid treated Nickel-Raney catalysts. The effects of reaction parameters such as reaction medium, temperature and hydrogen pressure were described. The acid treated Nickel-Raney catalyst exhibited the highest BAF yield in the THF-water mixed reaction medium. The relatively higher $\mathrm{Ni}^{0}$ species composition and larger surface area of the acid treated Nickel-Raney catalyst with specific reaction conditions contributed greatly to the BAF formation. The oligomeric species, such as furanic imine trimers and tetramers confirmed by MALDI-MS analysis were presented as the intermediates of DFF reductive amination.

\section{Keywords}

Reductive Amination, Primary Amine, 2,5-Bis(Aminomethyl)Furan, 2,5-Diformylfuran, Nickel Raney Catalyst

\section{Introduction}

Amines are important intermediates in the bulk and fine chemical industries including large-scale production of

${ }^{*}$ Corresponding author.

How to cite this paper: Le, N.-T., Byun, A., Han, Y., Lee, K.-I. and Kim, H. (2015) Preparation of 2,5-Bis(Aminomethyl)Furan by Direct Reductive Amination of 2,5-Diformylfuran over Nickel-Raney Catalysts. Green and Sustainable Chemistry, 5, 115127. http://dx.doi.org/10.4236/gsc.2015.53015 
numerous polymers. In addition, they represent interesting building blocks for the synthesis of pharmaceuticals, agrochemicals and food additives [1] [2]. Among the different types of amines, primary amines and di-amines are very useful intermediates for further derivatization and polymerization reactions [3]-[6]. In general, various types of amines have been prepared by the reductive amination of corresponding aldehyde compounds.

As shown in reaction Scheme 1, the reductive amination of 2,5-diformylfuran (DFF), a versatile platform chemical derived from catalytic oxidation of bio-mass based 5-hydroxymethylfurfural (HMF) [7]-[10] can lead to 2,5-bis(aminomethyl)furan (BAF), an useful di-amine compound. BAF has been considered as a new type of di-amine monomer for the production of polymers such as polyamides and polyurethanes [11]-[13].

However, the direct preparation of BAF from DFF has been not successful in spite of the recent progress of catalytic aminations. It requires the delicate approaches to prevent the formation of by-products of secondary, tertiary and polymeric amine species due to the condensation and/or hydrogenation of the reactive di-aldehyde groups and high nucleophilic amine groups of the product. Moreover, the bridge imine groups which are formed by the condensation of di-amine and di-aldehyde compounds need to be preserved during the reductive amination from the fast hydrogenation to minimize the polyamine type by-product formation [14]. The trans-imination of the bridge imine groups with ammonia has been known as an important reaction step for the selective formation of monomeric di-amine and di-imine species [14] [15]. Thus, the regulations of hydrogenation activity and trans-imination properties of the catalysts are of great importance in the reductive amination of very reactive di-aldehyde compound like DFF.

To the best of our knowledge, there has been no successful report showing the direct reductive amination of DFF to BAF using ammonia, even though other di-amine compounds, such as bis(aminomethyl)cyclohexane and 1,9-nonanediamine have been prepared from the corresponding dialdehydes, cyclohexanedicarboxaldehyde [14] and 1,9-nonanedial [16] [17]. Recently, Thomas et al. [18] claimed a two-step pathway to prepare BAF from DFF via furan-2,5-dicarbaldehyde dioxime formation by reacting with $\mathrm{NH}_{2} \mathrm{OH} \cdot \mathrm{HCl}(\mathrm{EtOH} 50 \%$ solvent, $50^{\circ} \mathrm{C}, 1 \mathrm{~h}$ ), followed by hydrogenation of the isolated dioxime over Nickel-Raney catalyst (THF solvent, 50 bar of $\mathrm{H}_{2}, 50^{\circ} \mathrm{C}, 12 \mathrm{~h}$ ) with an overall yield of $76 \%$.

In the present study, we report, for the first time, the direct reductive amination of DFF with ammonia to BAF with Nickel-Raney type catalysts under mild conditions. Herein, the reductive amination activity and selectivity variations of the Nickel-Raney catalyst and the acid treated Nickel-Raney catalyst are shown. Besides, the effects of key parameters and oligomeric imine forms of intermediates in the DFF amination are also described.

\section{Experimental}

\subsection{Catalyst Preparation}

In this research, Ni-Al alloy (Kanto Chemical Co., Inc., Japan) containing 50 wt\% of nickel and 50 wt\% of aluminum was used as a precursor to prepare Nickel-Raney catalysts for the reductive amination. This alloy was activated by alkaline leaching of aluminum using the well-known method [19]-[21]. Briefly, $1.0 \mathrm{~g}$ of Ni-Al alloy was slowly added to an excess of an aqueous $20 \mathrm{wt} \% \mathrm{NaOH}$ solution at $50^{\circ} \mathrm{C}$ under gentle stirring. After addition, the reaction system was kept on stirring $1 \mathrm{~h}$ for further alkali leaching. The black precipitate was washed with distilled water to neutrality, to remove alkali and aluminates then dried under nitrogen flow at $100^{\circ} \mathrm{C}$. Finally, the resulting catalyst (denoted as Ni-Raney) was immersed in the desired reaction medium to avoid contact with air prior to the subsequent catalytic amination.

The acid treated Nickel-Raney (AT-Ni-Raney) catalyst was prepared by a further treatment: The Ni-Raney catalyst suspension was acidified until $\mathrm{pH} 5$ by a dilute acetic acid solution, kept on stirring 30 min then washed with distilled water to neutrality, dried under nitrogen flow at $100^{\circ} \mathrm{C}$ and finally stored in the desired reaction medium.

For the comparison purpose, hydrogen peroxide treated AT-Ni-Raney $\left(\mathrm{H}_{2} \mathrm{O}_{2}\right.$-AT-Ni-Raney) catalyst was

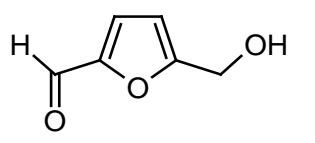

HMF
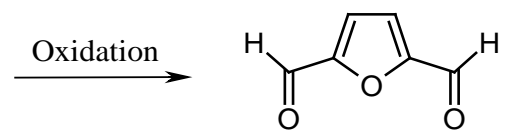

DFF

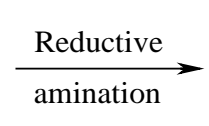

$$
\text { amination }
$$

Scheme 1. Formation and reductive amination of DFF.

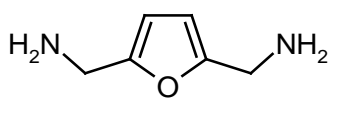

BAF 
prepared from the AT-Ni-Raney by treatment with an aqueous $0.1 \mathrm{wt} \% \mathrm{H}_{2} \mathrm{O}_{2}$ solution at room temperature for $30 \mathrm{~min}$, then washed with distilled water, dried under nitrogen and finally stored in the desired reaction medium.

\subsection{Reductive Amination}

Materials: DFF (>98\%, TCI, Japan), $\mathrm{H}_{2}$ (99.9\%, Deokyang, Korea), $\mathrm{NH}_{3}$ (99.9\%, Deokyang, Korea) and solvents (THF $\geq 99.9 \%$, Sigma-Aldrich; EtOH $\geq 99.9 \%$, Samchun; 1,4-Dioxane $\geq 99.8 \%$, Sigma-Aldrich) were obtained commercially and used as received.

In a typical reductive amination reaction, DFF (300 mg), catalyst (30 mg), internal GC standard and reaction medium (30 ml) were charged in the high pressure stainless steel reactor equipped with a magnetic stirring and a temperature control system. The air in the reactor was then replaced by hydrogen three times. Thereafter, liquid ammonia (2 ml) in pre-condensed form was introduced at room temperature. Finally, hydrogen was supplied to 10 bar total pressure and the reaction was started at the desired temperature. Liquid samples were taken from reactor during the course of the experiment to analyze conversion and yield. After $6 \mathrm{~h}$ of reaction, the reactor was cooled, the pressure was released and the catalyst was separated by centrifugation.

The conversion of DFF and the yield of BAF were determined by GC analysis with internal standard equipped with MEGA-1 column $(30 \mathrm{~m} \times 0.32 \mathrm{~mm})$ and flame ionization detector applying a temperature gradient from $60^{\circ} \mathrm{C}$ to $200^{\circ} \mathrm{C}$. The desired product was isolated after removal of solvent and purified by column chromatography (silica gel) method. The structure of BAF product was further confirmed by spectroscopies methods (see Figures S1-S3 of Supporting Information).

\subsection{Techniques}

Chemical analyses of catalysts were performed by the energy dispersive X-Ray fluorescence (EDXRF) using ARL QUANT'X/Thermo instrument equipped with an X-Ray tube of Rhodium and $\mathrm{Si}(\mathrm{Li})$ detector at energy resolution of $155 \mathrm{eV}$. The surface area measurements were calculated by the Brunauer, Emmett and Teller (BET) method on a Micromeritics ASAP 2040 system. Samples were transferred to the adsorption glass tube with the storage liquid and treated at $200^{\circ} \mathrm{C}$ under ultrahigh pure nitrogen flow for $2 \mathrm{~h}$ before measurement. The oxidation states of nickel and the surface composition of Ni-Raney catalysts were determined by X-Ray photoelectron spectroscopies (XPS) recording on KRATOS AXIS NOVA instrument.

\section{Results and Discussion}

\subsection{Catalyst Characterization}

The surface elemental compositions (wt\%) by EDXRF analysis and BET surface areas of the Ni-Raney type catalysts are first compared. As shown in Table 1, the surface composition of the total Ni species of the Ni-Raney catalyst is $61.8 \%$. After the acid treatment the total Ni content increases to $70.8 \%$ with decreasing the compositions of $\mathrm{Al}$ and $\mathrm{O}$ species. In addition, a large increase of surface area is obvious, changing from 12.2 $\mathrm{m}^{2} / \mathrm{g}$ to $44.7 \mathrm{~m}^{2} / \mathrm{g}$ which expands accessible reaction pathways to the $\mathrm{Ni}^{0}$ species of the Ni-Raney catalyst.

For the purpose of quantitative estimates of Ni components in the catalysts, XPS of Ni-Raney and AT-NiRaney samples were used. As can be seen from Figure 1, the Ni-Raney sample (black line) exhibits the presence of $\mathrm{Ni}$ metal as well as $\mathrm{Ni}$ compounds such as nickel oxide $\mathrm{NiO}$, nickel hydroxide $\mathrm{Ni}(\mathrm{OH})_{2}$ and nickel oxyhydroxide $\mathrm{NiOOH}$. The small peak centered at the binding energy value of $852.6 \mathrm{eV}$ is corresponding to $\mathrm{Ni}$

Table 1. Elemental composition (wt\%) of surface and surface area of catalysts.

\begin{tabular}{ccccc}
\hline Catalyst & Nickel $^{\mathrm{a}}$ & Aluminum $^{\mathrm{a}}$ & Oxygen $^{\mathrm{a}}$ & ${\text { Surface } \text { area }^{\mathrm{b}}}^{\mathrm{b}}$ \\
\hline Ni-Raney & 61.8 & 11.4 & 26.5 & 12.2 \\
AT-Ni-Raney & 70.3 & 8.5 & 21.0 & 44.7 \\
$\mathrm{H}_{2} \mathrm{O}_{2}$-AT-Ni-Raney & 64.2 & 10.5 & 24.9 & 45.3 \\
\hline
\end{tabular}

${ }^{a}$ Determined by EDXRF analysis; ${ }^{b}$ Measured at liq. $\mathrm{N}_{2}$ temperature, $\mathrm{m}^{2} / \mathrm{g}$ (BET method, after vacuum pre-treatment at $200^{\circ} \mathrm{C}$ for $\left.2 \mathrm{~h}\right)$. 


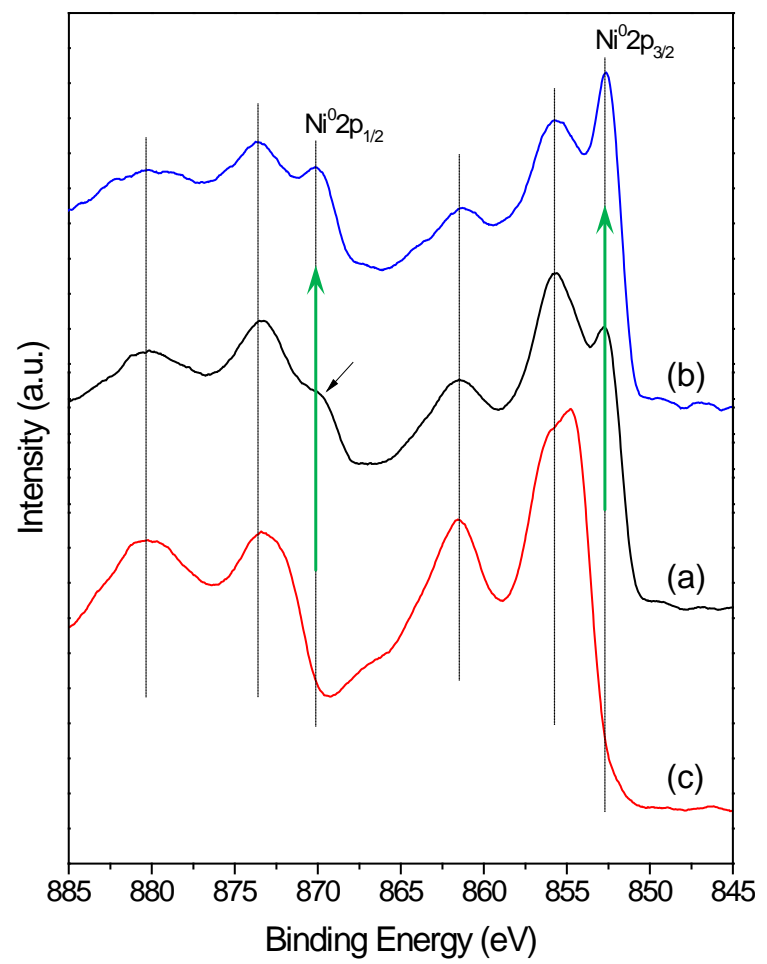

Figure 1. XPS results of (a) Ni-Raney catalyst, (b) ATNi-Raney catalyst, (c) $\mathrm{H}_{2} \mathrm{O}_{2}$-AT-Ni-Raney catalyst.

metal $2 \mathrm{p}_{3 / 2}$ species [22]-[24]. Ni metal component appears to have the additional contribution at $869.8 \mathrm{eV}$ (indicated by arrow) which is attributed to metal $2 \mathrm{p}_{1 / 2}$ species [23]. The contribution of $\mathrm{Ni}_{2} \mathrm{p}_{3 / 2}$ hydroxide and oxyhydroxide species are found at $855.7 \mathrm{eV}$ and $861.5 \mathrm{eV}$ while Ni2 $\mathrm{p}_{1 / 2}$ counterparts are located at $873.4 \mathrm{eV}$ and $880.2 \mathrm{eV}$. The peaks of $\mathrm{NiO}$ component are obscured by spectral overlap with that of $\mathrm{Ni}(\mathrm{OH})_{2}$. However, curve fitting procedures should be useful for a more meaningful interpretation for a series of similar Ni containing samples. The quantitative results for the full $2 \mathrm{p}\left(2 \mathrm{p}_{3 / 2}\right.$ and $\left.2 \mathrm{p}_{1 / 2}\right)$ of each component show the Ni being present as 17.5\% metal, 36.5\% oxide and $45.9 \%$ hydroxide and oxyhydroxide (Table 2). After the acid-treatment, however, the composition of $\mathrm{Ni}^{0}$ states in AT-Ni-Raney sample exhibits a significant increase, seen by the gain of intensity of peaks at $852.6 \mathrm{eV}$ and $869.8 \mathrm{eV}$ (Figure 1, blue line). Quantitative analysis of this spectrum derives the $\mathrm{Ni}^{0}$ composition of $37.7 \%$, which is much higher than that in case of the Ni-Raney catalyst. With $\mathrm{H}_{2} \mathrm{O}_{2}$-AT-NiRaney sample which shows completely oxidized states into oxide/oxyhydroxide species is distinguished by XPS analysis (Figure 1, red line and Table 2).

\subsection{Catalytic Activity}

The direct reductive amination scheme of DFF to BAF is depicted in Scheme 2. To avoid the complexity in the DFF reductive amination, a high molar ratio (30:1) of ammonia to DFF was applied in all experiments. The application of an excess amount of ammonia is a common technique to increase the selectivity to primary amine in the reductive amination of aldehydes [25].

A number of representative DFF reductive aminations results are shown in Table 3 for the Ni-Raney catalysts and other known precious metal amination catalysts. The full conversions of DFF were observed for all entries within $2 \mathrm{~h}$ due to the fast reaction of DFF with ammonia. The aminations of DFF with $\mathrm{Ru} / \mathrm{C}, \mathrm{Pt} / \mathrm{C}$ and $\mathrm{Pd} / \mathrm{CaCO}_{3}$ catalysts in ethanol, 1,4-dioxane and THF mediums including water added mediums of them gave no yield of BAF at various temperature and hydrogen pressure ranges (Entries 1-3). These behaviors are very different from the other aldehyde reductive aminations, because the catalysts containing metallic species of $\mathrm{Ru}, \mathrm{Pt}$ and Pd generally show the reductive amination activities for other aldehydes [26]-[28]. However, when applying the Ni-Raney and AT-Ni-Raney catalysts, we observed moderate range of BAF yields in the THF-water mixed 
Table 2. Quantitative analysis of Ni2p components present in Ni-Raney catalysts.

\begin{tabular}{cccc}
\hline \multirow{2}{*}{ Catalyst } & \multicolumn{3}{c}{ Component (\%) } \\
\cline { 2 - 4 } & $\mathrm{Ni}^{0}$ & $\mathrm{NiO}$ & $\mathrm{Ni}(\mathrm{OH})_{2}+\mathrm{NiOOH}$ \\
\hline Ni-Raney & 17.5 & 36.5 & 45.9 \\
AT-Ni-Raney & 37.7 & 24.1 & 38.2 \\
$\mathrm{H}_{2} \mathrm{O}_{2}$-AT-Ni-Raney & 0.0 & 40.3 & 59.7 \\
\hline
\end{tabular}

Table 3. Catalytic activity in the reductive amination of DFF.

\begin{tabular}{|c|c|c|c|c|c|}
\hline Entry & Catalyst & Solvent (vol\%) & $T\left({ }^{\circ} \mathrm{C}\right)$ & $P_{H 2}$ (bar) & BAF yield $^{\mathrm{a}}(\%)$ \\
\hline $1^{\mathrm{b}}$ & $\mathrm{Pt} / \mathrm{C}$ & Sol $^{e}$, Sol- $\mathrm{H}_{2} \mathrm{O}(90: 10)$ & 100,120 & 10 & - \\
\hline $2^{c}$ & $\mathrm{Pd} / \mathrm{CaCO}_{3}$ & Sol, Sol- $\mathrm{H}_{2} \mathrm{O}(90: 10)$ & 120 & 10,20 & - \\
\hline $3^{\mathrm{d}}$ & $\mathrm{Ru} / \mathrm{C}$ & Sol, Sol- $\mathrm{H}_{2} \mathrm{O}(90: 10)$ & 100,140 & 10 & - \\
\hline 4 & Ni-Raney & $\mathrm{EtOH}$ & 120,140 & $5,10,20$ & - \\
\hline 5 & Ni-Raney & $\mathrm{EtOH}-\mathrm{H}_{2} \mathrm{O}(90: 10)$ & 120 & 10,20 & - \\
\hline 6 & Ni-Raney & 1,4-Dioxane & 120 & 10 & - \\
\hline 7 & Ni-Raney & 1,4-Dioxane- $\mathrm{H}_{2} \mathrm{O}(90: 10)$ & 120 & 10 & - \\
\hline 8 & Ni-Raney & THF & 120 & 10,20 & - \\
\hline 9 & Ni-Raney & THF- $\mathrm{H}_{2} \mathrm{O}(90: 10)$ & 120 & 20 & 7.3 \\
\hline 10 & AT-Ni-Raney & THF & 120,140 & 20 & - \\
\hline 11 & AT-Ni-Raney & THF- $\mathrm{H}_{2} \mathrm{O}(95: 5)$ & 120 & 20 & 27.2 \\
\hline 12 & AT-Ni-Raney & THF- $\mathrm{H}_{2} \mathrm{O}(90: 10)$ & 100 & 20 & 28.3 \\
\hline 13 & AT-Ni-Raney & THF- $\mathrm{H}_{2} \mathrm{O}(90: 10)$ & 120 & 10 & 42.6 \\
\hline 14 & AT-Ni-Raney & THF- $\mathrm{H}_{2} \mathrm{O}(90: 10)$ & 120 & 5 & 18.1 \\
\hline 15 & $\mathrm{H}_{2} \mathrm{O}_{2}$-AT-Ni-Raney & THF- $\mathrm{H}_{2} \mathrm{O}(90: 10)$ & 120 & 20 & - \\
\hline
\end{tabular}

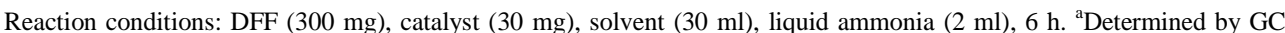
analysis with tetraethylene glycol dimethyl ether as the internal standard; ${ }^{\text {b,c,d }}$ Using $5 \mathrm{wt} \% \mathrm{Pt} / \mathrm{C}$ and $5 \mathrm{wt} \% \mathrm{Pd} / \mathrm{CaCO}_{3}$ (Strem Chemicals, USA), and $5 \mathrm{wt} \% \mathrm{Ru} / \mathrm{C}$ (Acros, Belgium) catalysts, respectively; ${ }^{\mathrm{e}}$ Sol = EtOH, 1,4-Dioxane, THF.<smiles>O=Cc1ccc(C=O)o1</smiles>

DFF

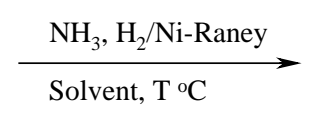

Solvent, $\mathrm{T}^{\circ} \mathrm{C}$

Scheme 2. Direct reductive amination of DFF to BAF.

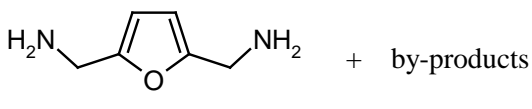

BAF

mediums, as shown in Entries 11-14. Without water addition to the reaction medium, both the Ni-Raney catalyst and the AT-Ni-Raney catalyst gave no BAF yield even at various amination conditions (Entries 8 and 10). The highest BAF yield of $42.6 \%$ for the AT-Ni-Raney catalyst which has higher surface area and $\mathrm{Ni}^{0}$ composition as shown in Table 1, Table 2 represents the close relationship between the BAF yield and $\mathrm{Ni}^{0}$ species in the catalyst surface.

It is very interesting to observe that BAF formation in the DFF reductive amination depends on the reaction medium, metal component and composition of the catalysts. To understand the key parameter of the DFF amination reaction, water content, reaction temperature, hydrogen pressure and reaction time effects were examined for the AT-Ni-Raney catalyst in THF-water mixed medium. As presented in Figure 2(a), the BAF yield increased with the addition of water. Without water addition, BAF formation was completely diminished, while 
the highest BAF yield (42.6\%) was shown in 10 volume\% of water-THF medium. At higher water ratio, up to 20 volume\%, BAF yield slightly decreased to 37\%. The effect of temperature on the BAF yield was studied in the temperature range of $100^{\circ} \mathrm{C}-140^{\circ} \mathrm{C}$. It is evident from Figure 2(b) that the BAF yield was largely affected by the reaction temperature changes. The BAF yield was found to be relatively lower at $100^{\circ} \mathrm{C}$. Raising the reaction temperature to $120^{\circ} \mathrm{C}$ showed a considerably positive effect on the formation of BAF. At $140^{\circ} \mathrm{C}$, however, only small increase of BAF yield was observed.

In addition to the water addition, reaction medium and reaction temperature, the hydrogen pressure and reaction time effects were examined. As illustrated in Figure 2(c), the initial increase in the hydrogen pressure from 5 to 10 bar markedly promoted the formation of BAF. Further increasing the pressure to 15 bar or even to 20 bar, the formation of BAF was not much affected. Reaction time for the reductive amination may depend on the other factors such as temperature and hydrogen pressure. The effects of reaction time with the evolution of reactants and products are shown in Figure 2(d). The complete DFF conversion was observed in $2 \mathrm{~h}$ and the BAF formation reached at the maximum yield after $5 \mathrm{~h}$ with the delay of $3 \mathrm{~h}$ from the complete DFF conversion. There was no change in BAF yield with reaction time after the maximum yield was reached.

The water addition effects to the reaction medium in the reductive amination of other type of aldehydes have been revealed in previous literatures. It has been demonstrated that the presence of a proper water concentration leads to a markedly lower tendency for the formation of undesired by-products and thus, to an increased selectivity to primary amines as described in the previous amination of 1,8-octanedial [15] and 1,9-nonanedial [16]. In the present study, the effect of the water addition on the BAF yield has been also found to be very crucial. To start and to promote the formation of BAF, the water addition to the THF medium was prerequisite with the
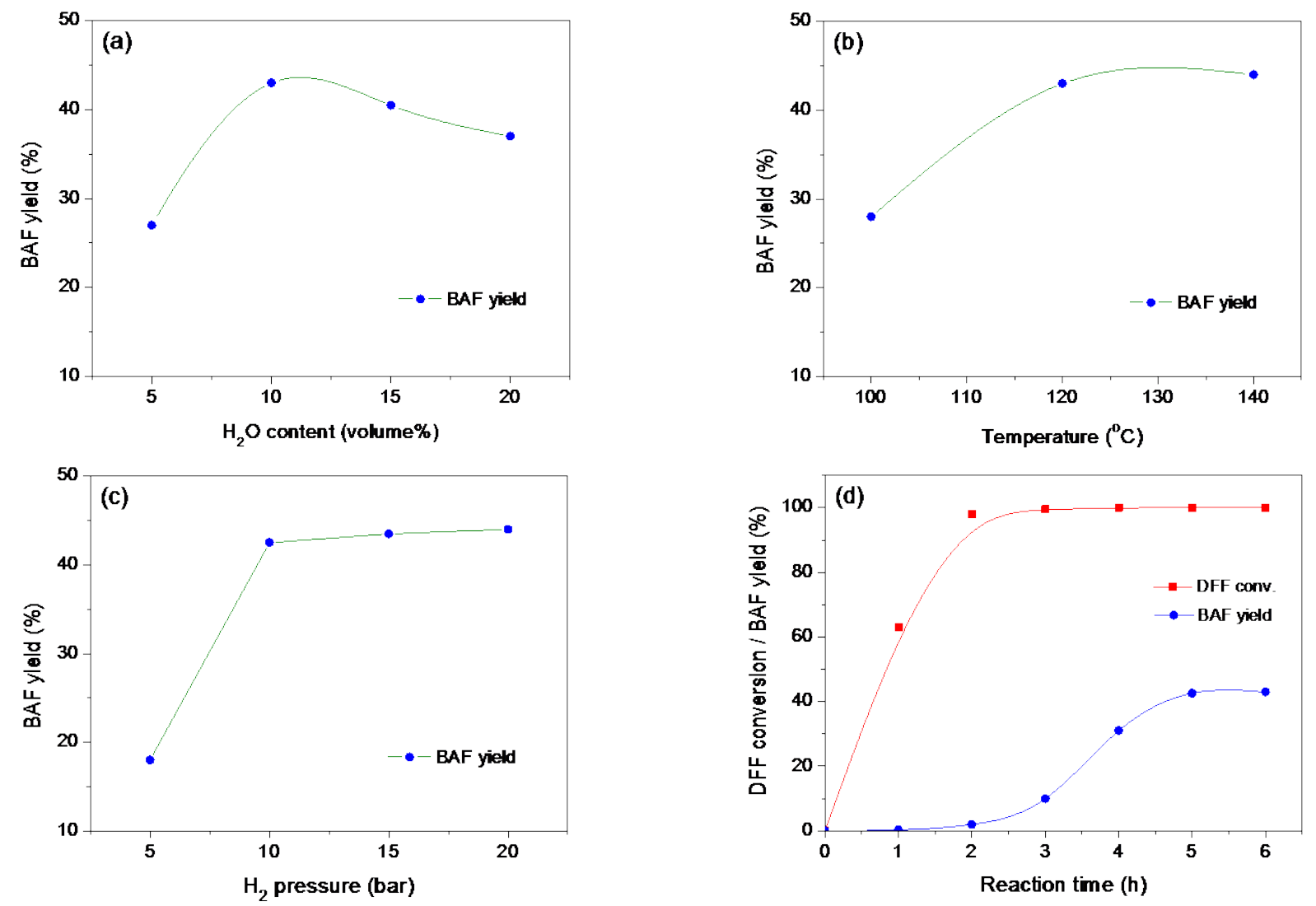

Figure 2. (a) Effect of water addition on BAF yield: DFF (300 mg), AT-Ni-Raney catalyst (30 mg), solvent (30 ml), liquid ammonia (2 ml), hydrogen (10 bar), $120^{\circ} \mathrm{C}, 6 \mathrm{~h}$; (b) BAF yield as a function of reaction temperature: DFF (300 mg), AT-Ni-Raney catalyst (30 mg), THF-water solvent (90:10, $30 \mathrm{ml})$, liquid ammonia (2 ml), hydrogen (10 bar), 6 h; (c) BAF yield as a function of hydrogen pressure: DFF (300 mg), AT-Ni-Raney catalyst (30 mg), THF-water solvent (90:10, $30 \mathrm{ml})$, liquid ammonia (2 ml), $120^{\circ} \mathrm{C}, 6 \mathrm{~h}$; (d) Evolution of DFF conversion and BAF yield with reaction time: DFF (300 mg), AT-Ni-Raney catalyst (30 mg), THF-water solvent (90:10, $30 \mathrm{ml})$, liquid ammonia (2 ml), hydrogen (10 bar), $120^{\circ} \mathrm{C}$. 
Ni-Raney type catalysts. With other metallic reductive amination catalyst components and mediums, BAF formations were not observed even with the water addition. The formation of primary diamine BAF in our amination system can be rationalized partially by the solvation effect of amine groups with water molecules, which may lower the condensation rate between aldehyde groups and the primary amine. In fact the primary alkylamines are considered as more basic than ammonia. With the presence of water in the reaction system in which primary alkylamine and ammonia are presented, alkylammonium ion is expected to be formed from primary amine with the chemical equilibrium. When primary diamine BAF is formed in the DFF reductive amination system, the following reaction is expected to form di-ammonium species (Scheme 3). The equilibrium effectively protects BAF from the condensations with dialdehyde DFF, since the alkylammonium ion of BAF is a no longer nucleophile.

As described above, the Ni-Raney catalysts are unique in the DFF reductive amination to BAF in the THFwater medium. The effectiveness of the Ni-Raney catalysts can be attributed to the proper and selective hydrogenation ability toward the imine groups of different types. The two types of imine groups are expected to be formed in the early stage of amination reaction by the imination of DFF as described in Scheme 4: The terminal imine groups which are attached to the furan ring and the bridge type internal imine groups which connect two furan moieties. In the reaction system, the hydrogenation activity of the Ni-Raney catalysts, compared with other precious metal amination catalysts, may catalyze selectively the terminal imine hydrogenation to form BAF preferably rather than the internal type imine hydrogenation to leave the internal imines for the trans-imination. The difference in the hydrogenation activities of Ru and Pd catalysts for the two types of imines was also proposed to explain the product selectivity variations of benzaldehyde reductive amination [27].

Regarding the reaction intermediates involved in the DFF reductive amination, we note that the full DFF conversion is achieved quickly while only a small amount of BAF appeared in the early stage of amination as shown in Figure 2(d). It means that DFF is converted to certain intermediates before the final BAF formation, most probably the imine type oligomeric furan species, because DFF and corresponding diimine and diamine (BAF) are very liable to condense to oligomeric or polymeric furan imine species. In the case of reductive amination of mono-aldehyde such as benzaldehyde, the internal imine intermediate was appeared predominantly before the product benzylamine formation [27]. GC analysis of the reaction mixture shows no formation of 5-hydroxymethylfurfural and 2,5-bis(hydroxymethyl)furan which are derived from the partial and total hydrogenation of DFF. The oligomeric intermediates during the DFF reductive amination were confirmed by MALDI-MS analysis. We separated a brown precipitate of strong imine stretching vibrations by IR spectroscopy (Figure S4 of Supporting Information) and of molecular weight mainly of $\sim 349$ and $\sim 428$ that could be designated to furan imine trimer and tetramer species, respectively (Figure S5(a) of Supporting Information). We also confirmed the presence of both macrocyclic oligomers and linear ones with molecular weights range of 430 to 1050 in the reaction medium which are in good agreement with the oligomeric furan moiety forms (Figure S5(b) of Supporting Information). Interestingly, the brown precipitate and oligomers were disappeared at the end of the amination reactions which supports that the oligomeric imine species were converted to the BAF and<smiles>C=C[C@H](O)Cc1ccc(C[NH3+])o1</smiles>

Scheme 3. BAF in equilibrium with water, leading to di-ammonium species.
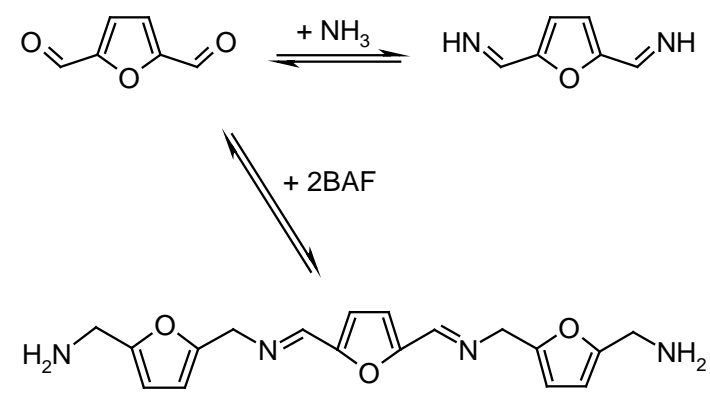

Scheme 4. Imination of DFF to two types of imine groups. 
unidentified by-products. In the case of reductive amination of cyclohexanedicarboxaldehyde, similar type intermediate of macrocyclic polyimine of $\sim 494$ molecular weight was observed that corresponds to a cyclic intermediate of two molecules of the cyclohexanedicarboxaldehyde (A) with two molecules of the product diamine (B). This $A_{2} B_{2}$ oligomer was tended to be the most prevalent species. Besides, the other cyclic $A_{3} B_{3}, A_{4} B_{4}, A_{5} B_{5}$ species and a series of linear species having molecular weight up to about 1000 were also detected [14].

Now, we discuss a reaction scheme for the formation of BAF from DFF by adopting the effects of reaction parameters, the proper hydrogenation activity of the Ni-Raney catalysts and the imine oligomeric intermediates of the DFF reductive amination system. In the reaction scheme, as shown in Figure 3, the two aldehyde groups of DFF first reacts with ammonia to form terminal diimines with equilibrium which then are hydrogenated to BAF over the Ni-Raney catalysts. DFF, diimine and BAF in the reaction system condense to form cyclic trimer, tetramer, linear oligomeric and polymeric imine species. These imine intermediates further undergo the transimination with ammonia to form terminal furanic diimine and BAF, followed by hydrogenation of the furanic diimine which affords BAF (route A). The oligomeric and polymeric imine intermediates can be transformed by the direct hydrogenation to macrocyclic and linear amines (routes B and C) that is related with the by-product formations. It may be also suggested in the reaction scheme that both the suppression of the internal imine species formations in the THF-water mixed medium and the proper regulation of the internal imine group hydrogenation activity by the Ni-Raney catalysts which reduce by-product formation play main roles to promote the direct reductive amination of DFF to BAF.

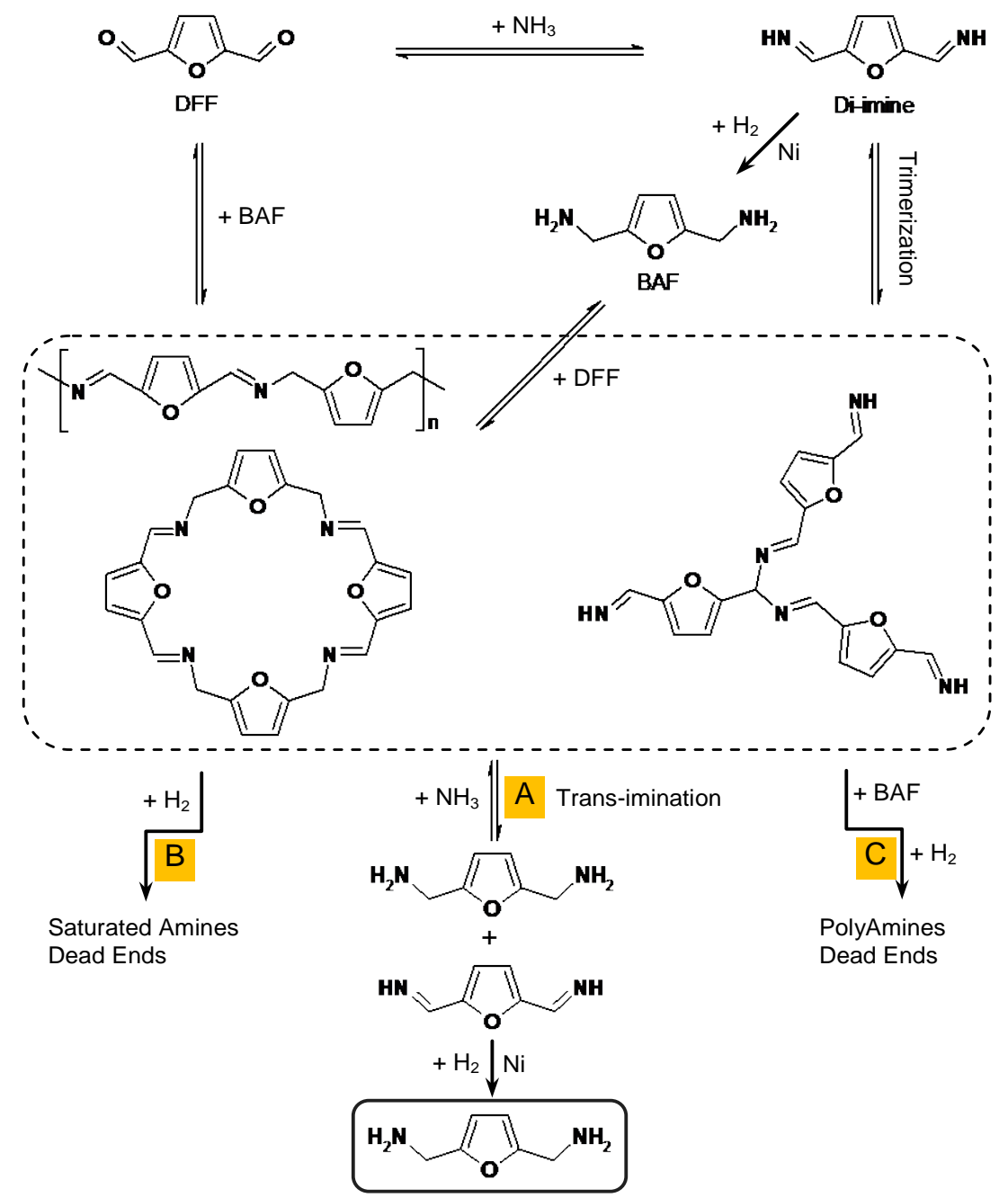

Figure 3. Proposed reaction intermediates for the formation of BAF with macrocyclic and linear polymers during the reductive amination of DFF. 


\section{Conclusion}

In conclusion, 2,5-bis(aminomethyl)furan was prepared by the direct reductive amination of 2,5-diformylfuran with ammonia in one step approach. The Ni-Raney catalysts showed reductive amination activities in THFwater mixed medium. The BAF yield of $42.6 \%$ was achieved when the acid treated Ni-Raney catalyst was applied under the optimal reaction conditions. It is proposed that the combination of THF-water mixed medium and the $\mathrm{Ni}^{0}$ component of the Ni-Raney catalysts plays a crucial role in the DFF reductive amination to BAF. Moreover, XPS, BET surface area and EDXRF analysis also support that the BAF formation is related with the $\mathrm{Ni}^{0}$ component of the Ni-Raney catalysts. The oligomeric imine trimer and tetramer species, confirmed by MALDI-MS analysis, are suggested as main intermediates in the direct reductive amination of DFF to BAF.

\section{Acknowledgements}

The authors acknowledge the Korea Research Institute of Chemical Technology (KRICT), Daejeon, Korea for supporting this work.

\section{References}

[1] Wittcoff, H.A., Reuben, B.G. and Plotkin, J.S. (2004) Industrial Organic Chemicals. 2nd Edition, Wiley-Interscience, New York. http://dx.doi.org/10.1002/0471651540

[2] Hartwig, J.F. (2002) Handbook of Organo-Palladium Chemistry for Organic Synthesis. Vol. 1, Wiley-Interscience, New York, 1051. http://dx.doi.org/10.1002/0471212466.ch42

[3] Kreye, O., Mutlu, H. and Meier, M.A.R. (2013) Sustainable Routes to Polyurethane Precursors. Green Chemistry, 15, 1431-1455. http://dx.doi.org/10.1039/c3gc40440d

[4] Roesky, P.W. (2009) Catalytic Hydroaminoalkylation. Angewandte Chemie International Edition, 48, 4892-4894. http://dx.doi.org/10.1002/anie.200900735

[5] Kruger, K., Tillack, A. and Beller, M. (2009) Recent Innovative Strategies for the Synthesis of Amines: From C-N Bond Formation to C-N Bond Activation. ChemSusChem, 2, 715-719. http://dx.doi.org/10.1002/cssc.200900121

[6] Muller, T.E., Hultzsch, K.C., Yus, M., Foubelo, F. and Tada, M. (2008) Hydroamination: Direct Addition of Amines to Alkenes and Alkynes. Chemical Reviews, 108, 3795-3892. http://dx.doi.org/10.1021/cr0306788

[7] Le, N.T., Lakshmanan, P., Cho, K., Han, Y. and Kim, H. (2013) Selective Oxidation of 5-Hydroxymethyl-2-Furfural into 2,5-Diformylfuran over $\mathrm{VO}^{2+}$ and $\mathrm{Cu}^{2+}$ Ions Immobilizedon Sulfonated Carbon Catalysts. Applied Catalysis A: General, 464-465, 305-312. http://dx.doi.org/10.1016/j.apcata.2013.06.002

[8] Yang, Z.Z., Deng, J., Pan, T., Guo, Q.X. and Fu, Y. (2012) A One-Pot Approach for Conversion of Fructose to 2,5Diformylfuran by Combination of $\mathrm{Fe}_{3} \mathrm{O}_{4}-\mathrm{SBA}-\mathrm{SO}_{3} \mathrm{H}$ and K-OMS-2. Green Chemistry, 14, 2986-2989. http://dx.doi.org/10.1039/c2gc35947b

[9] Takagaki, A., Takahashi, M., Nishimura, S. and Ebitani, K. (2011) One-Pot Synthesis of 2,5-Diformylfuran from Carbohydrate Derivatives by Sulfonated Resin and Hydrotalcite-Supported Ruthenium Catalysts. ACS Catalysis, 1, $1562-$ 1565. http://dx.doi.org/10.1021/cs200456t

[10] Ma, J., Du, Z., Xu, J., Chu, Q. and Pang, Y. (2011) Efficient Aerobic Oxidation of 5-Hydroxymethylfurfural to 2,5Diformylfuran, and Synthesis of a Fluorescent Material. ChemSusChem, 4, 51-54. http://dx.doi.org/10.1002/cssc.201000273

[11] Gandini, A. and Belgacem, M.N. (1997) Furans in Polymer Chemistry. Progress in Polymer Science, 22, $1203-1379$. http://dx.doi.org/10.1016/S0079-6700(97)00004-X

[12] Moreau, C., Belgacem, M.N. and Gandini, A. (2004) Recent Catalytic Advances in the Chemistry of Substituted Furans from Carbohydrates and in the Ensuing Polymers. Topics in Catalysis, 27, 11-30. http://dx.doi.org/10.1023/B:TOCA.0000013537.13540.0e

[13] Lichtenthaler, F.W. (2002) Unsaturated $O$ - and $N$-Heterocycles from Carbohydrate Feedstocks. Accounts of Chemical Research, 35, 728-737. http://dx.doi.org/10.1021/ar010071i

[14] Molitor, E.J. and Toyzan, T.W. (2008) Process for the Reductive Amination of Aldehydes and Ketones. WO Patent No. 076795A1.

[15] Kampmann, D., Weber, J. and Kniep, C. (1991) Process for the Preparation of Diamines. US Patent No. 5055618.

[16] Nagareda, K., Tokuda, Y. and Suzuki, S. (1998) Process for Producing Diamines from Dialdehydes. European Patent No. 0878462A1.

[17] Tokuda, Y. and Suzuki, S. (2003) Process for Producing Diamines from Dialdehydes. European Patent No. 
1348688A1.

[18] Haas, T., Tacke, T., Pfeffer, J.C., Klasovky, F., Rimbach, M., Volland, M. and Ortelt, M. (2012) Process for Producing 2,5-Diformylfuran and Derivatives thereof. WO Patent No. 004069A1.

[19] Devred, F., Hoffer, B.W., Sloof, W.G., Kooyman, P.J., van Langeveld, A.D. and Zandbergen, H.W. (2003) The Genesis of the Active Phase in Raney-Type Catalysts: The Role of Leaching Parameters. Applied Catalysis A: General, 244, 291-300. http://dx.doi.org/10.1016/S0926-860X(02)00601-4

[20] Fouilloux, P. (1983) The Nature of Raney Nickel, Its Adsorbed Hydrogen and Its Catalytic Activity for Hydrogenation Reactions (Review). Applied Catalysis, 8, 1-42. http://dx.doi.org/10.1016/0166-9834(83)80051-7

[21] Zhang, L., Lu, Q., Hou, Y. and Ying, W. (2013) Synthesis of an Amine Terminated Polyether: Effects of the Activation Conditions on a Raney Nickel Catalyst. Reaction Kinetics Mechanism and Catalysis, 108, 139-149. http://dx.doi.org/10.1007/s11144-012-0507-4

[22] Biesinger, M.C., Payne, B.P., Grosvenor, A.P., Lau, L.W.M., Gerson, A.R. and Smart, R.S.C. (2011) Resolving Surface Chemical States in XPS Analysis of First Row Transition Metals, Oxides and Hydroxides: Cr, Mn, Fe, Co and Ni. Applied Surface Science, 257, 2717-2730. http://dx.doi.org/10.1016/j.apsusc.2010.10.051

[23] Moulder, J.F., Stickle, W.F., Sobol, P.E. and Bomben, K.D. (1995) Handbook of X-Ray Photoelectron Spectroscopy. Physical Electronics Inc., Chanhassen.

[24] Biesinger, M.C., Payne, B.P., Lau, L.W.M., Gerson, A. and Smart, R.S.C. (2009) X-Ray Photoelectron Spectroscopic Chemical State Quantification of Mixed Nickel Metal, Oxide and Hydroxide Systems. Surface and Interface Analysis, 41, 324-332. http://dx.doi.org/10.1002/sia.3026

[25] Gomez, S., Peters, J.A. and Maschmeyer, T. (2002) The Reductive Amination of Aldehydes and Ketonesand the Hydrogenation of Nitriles: Mechanistic Aspects and Selectivity Control. Advanced Synthesis and Catalysis, 344, 10371057.

[26] Qi, F., Hu, L., Lu, S., Cao, X. and Gu, H. (2012) Selective Synthesis of Secondary Amines by Pt Nanowire Catalyzed Reductive Amination of Aldehydes and Ketones with Ammonia. Chemical Communication, 48, 9631-9633. http://dx.doi.org/10.1039/c2cc34461k

[27] Gomez, S., Peters, J.A., van der Waal, J.C., van den Brink, P.J. and Maschmeyer, T. (2004) The Rationalization of Catalyst Behavior in the Reductive Amination of Benzaldehyde with Ammonia Using a Simple Computer Model. Applied Catalysis A: General, 261, 119-125. http://dx.doi.org/10.1016/j.apcata.2003.10.037

[28] Bagal, D.B., Watile, R.A., Khedkar, M.V., Dhake, K.P. and Bhanage, B.M. (2012) PS-Pd-NHC: An Efficient and Heterogeneous Recyclable Catalyst for Direct Reductive Amination of Carbonyl Compounds with Primary/Secondary Amines in Aqueous Medium. Catalysis Science and Technology, 2, 354-358. http://dx.doi.org/10.1039/C1CY00392E 


\section{Supporting Information}

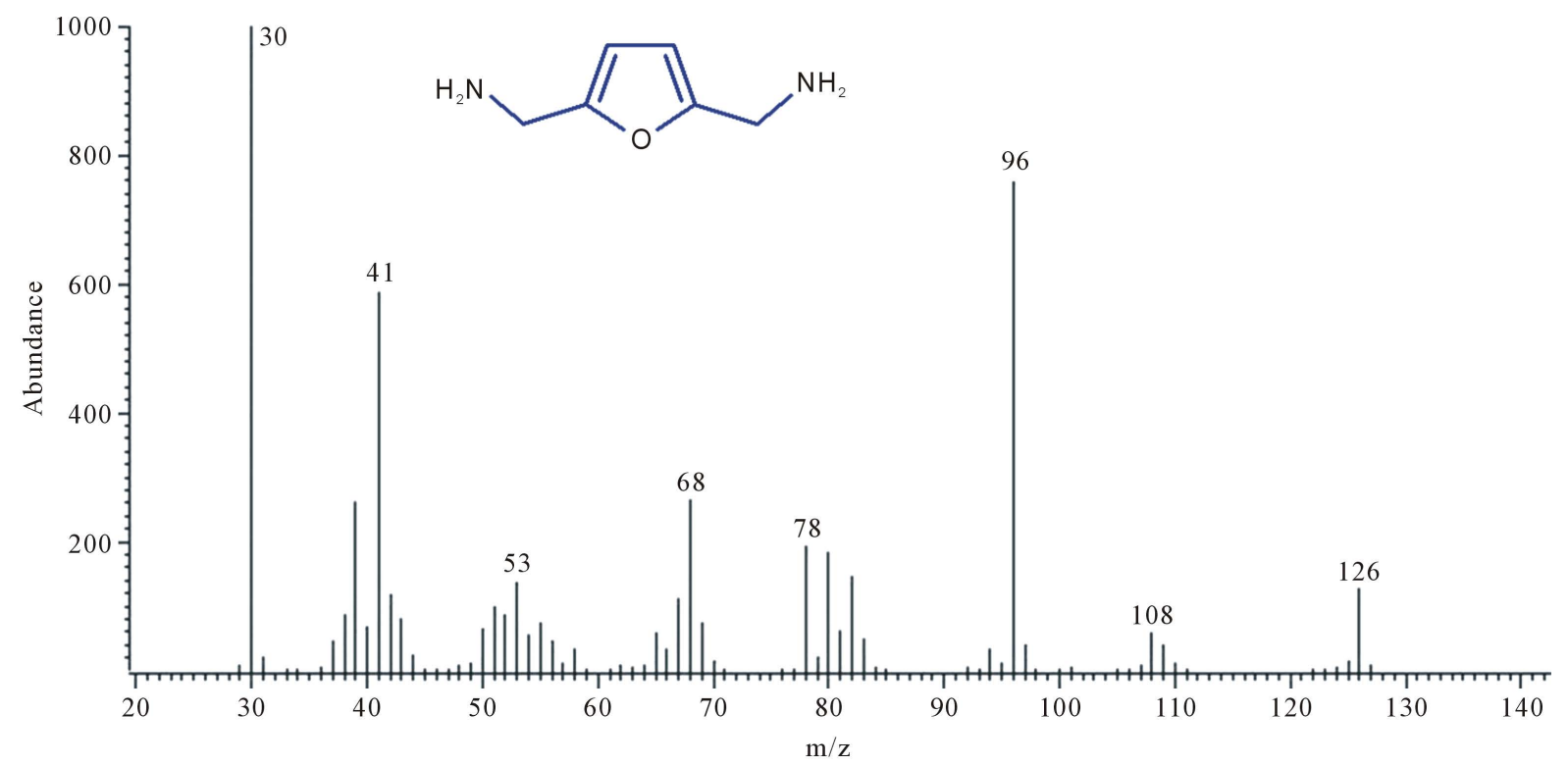

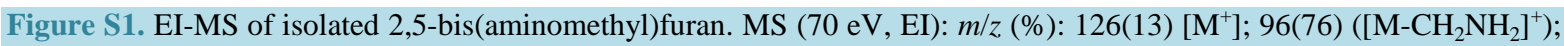
68(27) $\left(\left[\mathrm{M}-\mathrm{CH}_{2} \mathrm{NH}_{2}-\mathrm{CO}\right]^{+}\right)$.
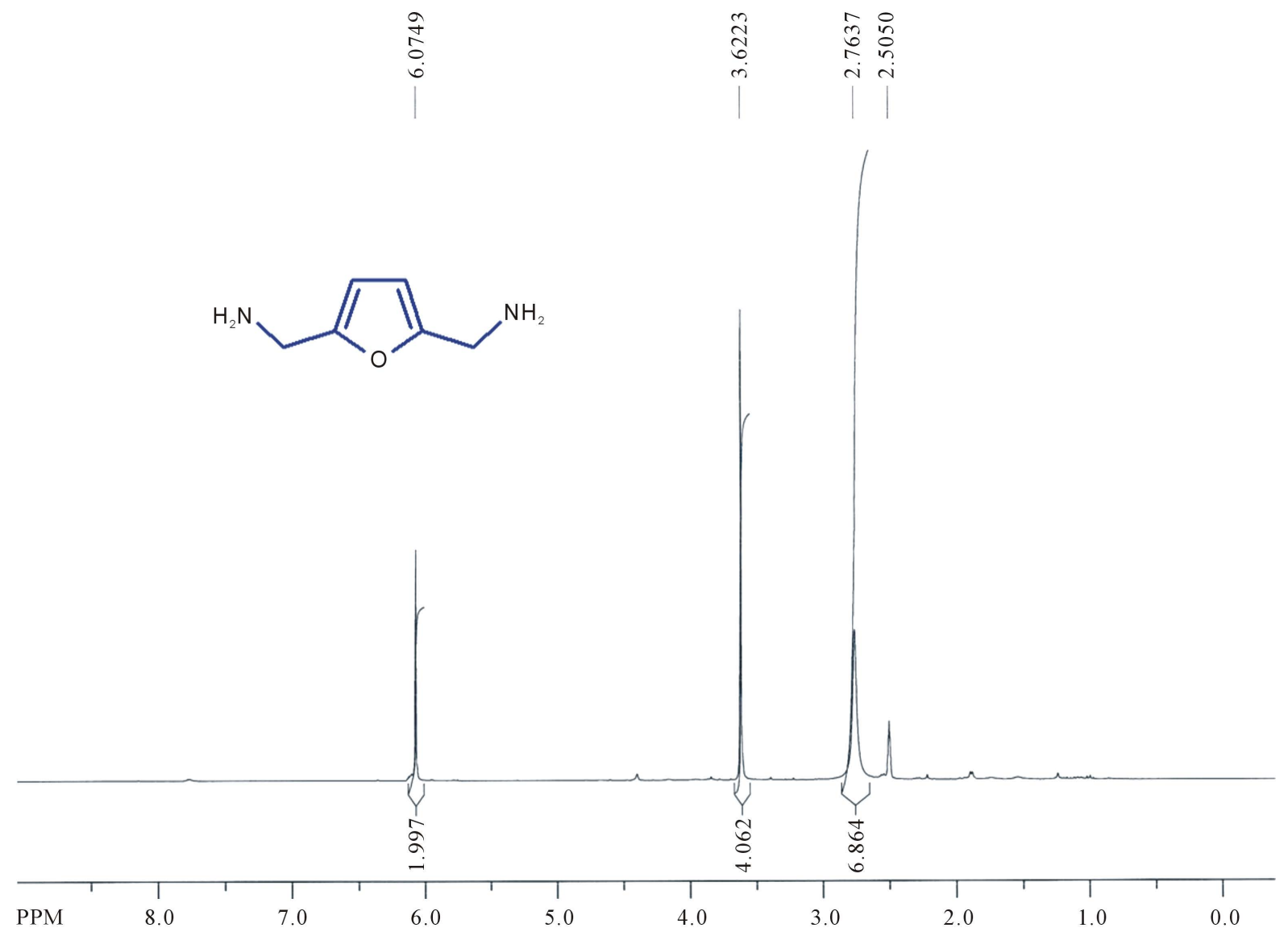

Figure S2. ${ }^{1} \mathrm{H}-\mathrm{NMR}\left(\mathrm{DMSO}-\mathrm{d}_{6}\right.$ ) of isolated 2,5-bis(aminomethyl)furan. ${ }^{1} \mathrm{H}-\mathrm{NMR}\left(300 \mathrm{MHz}, \mathrm{DMSO}-d_{6}\right): \delta(\mathrm{ppm})=2.7(\mathrm{~s}$, $6 \mathrm{H}$, including $4 \mathrm{H}$ of $\mathrm{NH}_{2}$ and $2 \mathrm{H}$ of $\left.\mathrm{H}_{2} \mathrm{O}\right) ; 3.6\left(\mathrm{~s}, 4 \mathrm{H}, \mathrm{CH}_{2}\right) ; 6.0(\mathrm{~s}, 2 \mathrm{H}$, furan). 


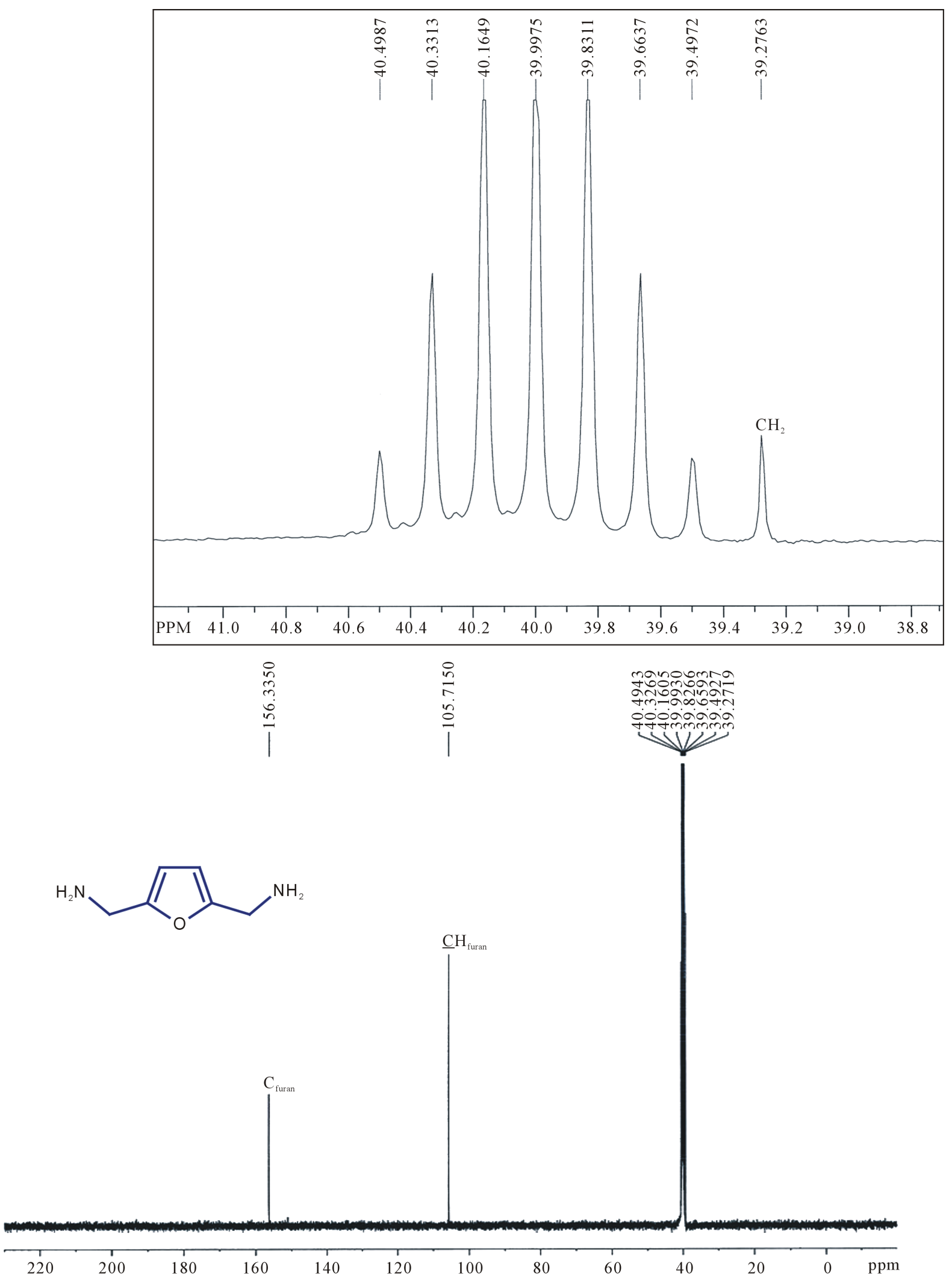

Figure S3. ${ }^{13} \mathrm{C}-\mathrm{NMR}$ (DMSO- $d_{6}$ ) of isolated 2,5-bis(aminomethyl)furan. ${ }^{13} \mathrm{C}-\mathrm{NMR}\left(500 \mathrm{MHz}\right.$, DMSO- $\left.d_{6}\right): \delta(\mathrm{ppm})=39.2$ (s, $\underline{\mathrm{CH}}_{2}$ ); 105.7 (d, $\underline{\mathrm{CH}}$, furan); 156.3 (s, C, furan). 


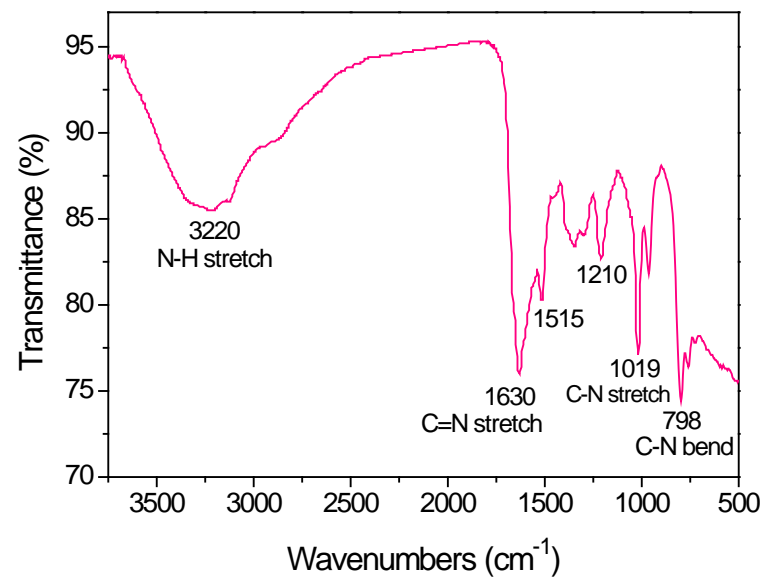

Figure S4. IR spectrum of brown precipitate.
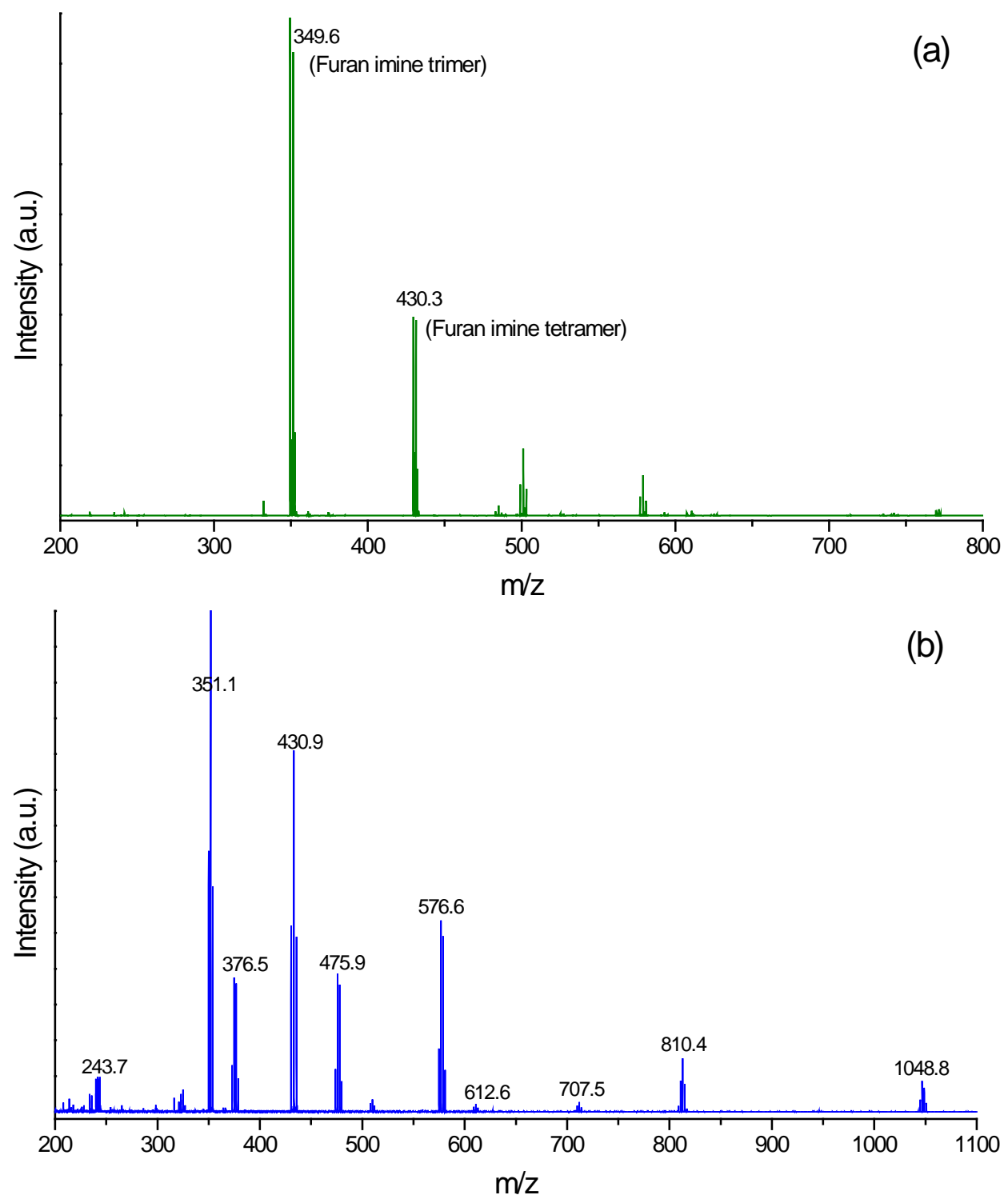

Figure S5. MALDI-MS of (a) brown precipitate and (b) reaction solution. 